\title{
Clogging of granular material in vertical pipes discharged at constant velocity
}

\author{
Diego López-Rodríguez ${ }^{1, \star}$, Iker Zuriguel ${ }^{1, \star \star}$ and Diego Maza ${ }^{1, \star \star \star}$ \\ ${ }^{1}$ Universidad de Navarra (Spain)
}

\begin{abstract}
We report an experimental study on the flow of spherical particles through a vertical pipe discharged at constant velocity by means of a conveyor belt placed at the bottom. For a pipe diameter 3.67 times the diameter of the particles, we observe the development of hanging arches that stop the flow as they are able to support the weight of the particles above them. We find that the distribution of times that it takes until a stable clog develops, decays exponentially. This is compatible with a clogging probability that remains constant during the discharge. We also observe that the probability of clogging along the pipe decreases with the height, i.e. most of the clogs are developed near the bottom. This spatial dependence may be attributed to different pressure values within the pipe which might also be related to a spontaneous development of an helical structure of the grains inside the pipe.
\end{abstract}

\section{Introduction}

In mining, it is quite frequent that the transport of rocks and ore between different levels is done through underground excavated tubes or ore passes [1]. This method has been traditionally employed in underground mining, but nowadays it is becoming extended to open-cast mining. The reasons are of different nature and transcendence, ranging from economical to environmental. Indeed, using gravity driving force to deliver material trough a pipe supposes important savings in the economical costs related to the transportation with trucks through the typically long and winding roads that go from the top of the mine to the bottom. In addition, dust production is considerably reduced when comparing pipe transport with the traditionally implemented strategy of throwing the material from one bench to the other. Other advantages of this pipe transport system are lessening the visual impact of traditional open-cast mines and reducing the noise levels in nearby populated areas. Apart from transport, ore passes also serve for low cost storage which is essential to efficient mine operation.

A typical ore pass has three basic parts: (1) the pipe itself connecting two or more levels in a mine; (2) the topend facilities to incorporate material, for example diggers, trucks and (sometimes) crushers; (3) bottom-end structures such as crushers and belts which are necessary to control material extraction. The bad operation of any of the parts is critical to the economic performance of mining operations.

Despite its great potential, the design of ore passes do not seem to receive the same engineering attention as

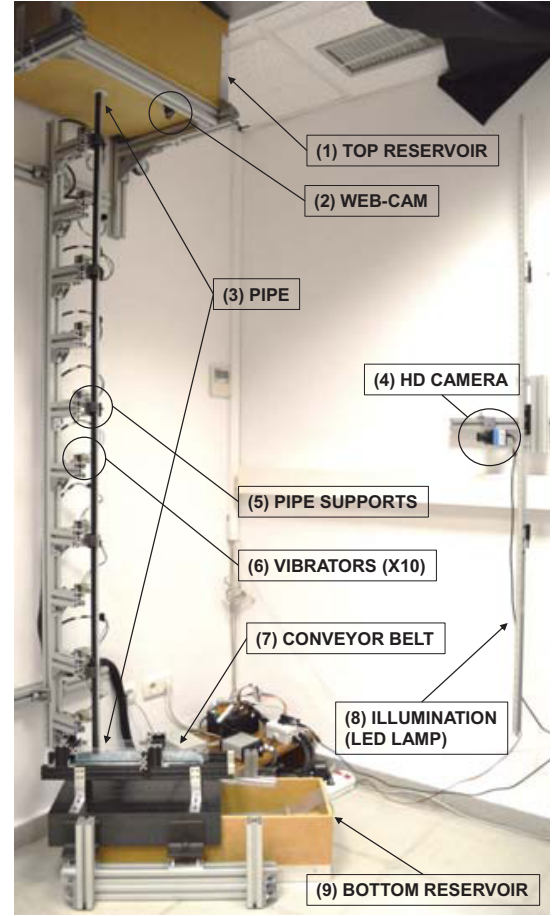

Figure 1. Experimental setup. In the image: (1) top reservoir from which the pipe is filled; (2) web-cam used to determine the time at which a clog occurs; (3) two meters long transparent pipe; (4) camera that takes pictures of the entire pipe; (5) pipe holders; (6) 10 vibrators controlled independently used to break the clogs; (7) conveyor belt that extracts the granular material; (8) lamp led; (9) bottom reservoir.

\footnotetext{
${ }^{\star}$ e-mail: dlopez.14@alumni.unav.es

$\star \star$ e-mail: iker@unav.es

$\star \star \star$ e-mail: dmaza@unav.es
} 
other mining infrastructures such as silos. Indeed, from the scarce works available [2-4] it becomes evident that many different aspects should be considered. For example, choosing the inclination of ore passes -which typically ranges from vertical to $30^{\circ}$ - is a practice based in a few empirical observations. The cross sections may be square, rectangular or circular and the diameter (or the side length) should be large enough to guarantee the absence of hangups. These hang-ups are typically caused by the formation of a stable structure of coarse material and are, by far, the most important problem associated to this, otherwise, convenient method to transport raw material in mines [4].

The development of clogs that cause arrest of the flow has been widely studied for the case of silos after the original work of K. To et al. [5]. Typically, clogging in silos is characterized by means of the avalanche size, defined as the number of beads that flow out of the silo from the destruction of a clogging arch until the development of a new one. We know that, for a given outlet and particle size, the avalanche size distribution is an exponential tail [6-9] suggesting that the probability of clogging remains constant over the whole avalanche duration. Also, it is known that the avalanche size grows very quickly with the outlet size. In this sense, although the existence of a critical outlet size above which clogging may never occur was originally proposed [7], further works have suggested that this transition does not necessarily exist $[8,9]$.

Going back to the pipe flow case, Janda et al. [10] experimentally evidenced the development of clogs in an inclined pipe full of a non-spherical ballast of limestone that was discharged at constant speed by means of a belt at the bottom. Their findings are compatible with a clogging probability that remains constant during the discharge. In addition, they show that the amount of material discharged before the development of a clog increases as the tube diameter is enlarged, the velocity of the conveyor belt grows, and the tube tilt deviates from the vertical. In another work, Verbücheln et al. [11] reported a numerical work where clogging was observed in a system of spheres falling freely under the action of gravity within a confined cylinder with periodic boundary conditions in the direction of gravity. At the beginning, grains accelerated but eventually, a limit velocity was achieved due to collisions. Then, depending on the ratio between the pipe diameter and the beads, the flow became intermittent giving rise to clogs. Interestingly, they show that inducing very well ordered patterns by means of the introduction of an inner helical texture in the pipe leads to a notable reduction of clogging.

In this work we design a experimental setup to analyze in detail the phenomenon of clogging in a vertical tube. First, we will show that clogging of spheres in perfectly vertical tubes is possible, an issue that has been only observed in numerical simulations [11]. Then, we will study both the temporal and spatial dependence of the clogging development. We are not aware of any work that characterizes the position at which clogs occur along the pipe and, as an improvement from previous work [10], we have implemented a system that allows this kind of measurement.

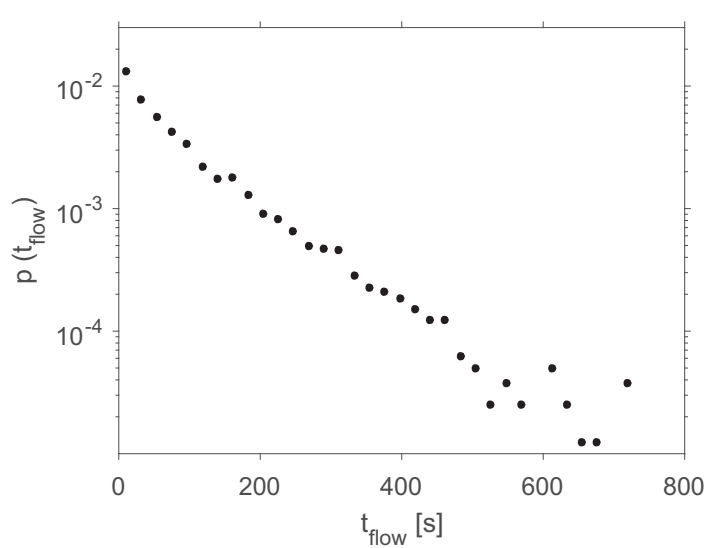

Figure 2. Probability distribution of avalanche duration. Note the semi-logarithmic scale.

\section{Experimental setup}

The experimental setup (Fig. 1) consisted of a long narrow pipe full of grains which were extracted at a constant rate by means of a conveyor belt placed at the bottom of the pipe. The granular material was formed by mono-disperse spherical glass beads of $0.6 \mathrm{~cm}$ diameter. The pipe was made of transparent methacrylate to allow the observation of clogs. The inner diameter was $2.2 \mathrm{~cm}$ which supposes a ratio between pipe and particle diameters of 3.67. The pipe had a length of $200 \mathrm{~cm}$ and the inclination with respect the horizontal was $90^{\circ}$, i.e. it was vertically oriented. The supporting structure was made of aluminum profiles fixed to the wall of the laboratory in order to prevent any movement or vibration.

The material was extracted with a conveyor belt perpendicular to the pipe. The gap between the pipe exit and the belt was $1 \mathrm{~cm}$. This configuration avoids the formation of clogs between the end of the pipe and the surface of the belt. The width of the belt, $6 \mathrm{~cm}$, and the distance from the end of the pipe to the rear part of the belt, $6 \mathrm{~cm}$, were kept constant.

The velocity of the belt, which determines the flowrate, was controlled by digital hardware allowing a straightforward modification of this parameter. The velocity was set at $v=0.215 \mathrm{~cm} / \mathrm{s}$ in all the experiments shown here. At the end of the conveyor, the granular material is accumulated in a box with capacity for the $10^{5}$ particles of glass beads employed (25 kilograms). Once all the beads have flown out of the pipe, they are poured in another reservoir of similar size placed at the top of the pipe. From there, the material is refilled into the pipe through an orifice of the same diameter than the pipe. As the ratio between the pipe diameter and the particle size is 3.67, clogs appear rather frequently at that top bottleneck [6]. To prevent such clogs, we build an autonomous system controlled by an Arduino $\AA$ consisting on an inverted cone that moves up and down with an amplitude of $5 \mathrm{~cm}$ in a period of 3 seconds. This device was placed inside the top reservoir, just above the orifice, hence leading to the breakage 
of all the arches formed at that upper orifice and allowing a constant refilling of the pipe.

The measuring protocol was: first, the pipe was filled through the reservoir at the top. Then, the conveyor was switched on and the material started to flow through the pipe by gravity. This instant was registered and defined the beginning of the avalanche, $t_{\text {start }}$. When eventually, a hanging arch clogged the pipe at any position, all the material above it stopped. This precise moment was detected by means of a web-cam, placed at the top of the pipe which performed a real-time analysis of a $3.5 \mathrm{~cm}$ height region. We compared the pixel values of two consecutive frames (every second) and we decided that a clog exist whenever these two images were identical. To ensure that the clog was stable we continued extracting material during the time that the conveyor needs to transport the distance of two pipe diameters. Therefore, we defined the duration of an avalanche: $t_{\text {flow }}=t_{\text {end }}-t_{\text {start }}$, where $t_{\text {end }}$ is the time defined by the two equal consecutive images.

A second camera (1600 $\times 1200$ pixels resolution and 15 frames per second) recorded the entire pipe with the objective of finding the position of the clog, $h_{c l o g}$ with respect to the bottom of the pipe. To this end, we analyzed the value of the pixels in a rectangular region of 8 pixels width and almost 1600 high (spanning the whole length of the pipe). The regions of the pipe with and without granular material were easily identified because, under proper illumination, beads were shown as white pixels, whereas empty regions appeared as black. We define the positions of the clogs at these places where in a slice of $1.2 \mathrm{~cm}$ height, all the pixels were black.

After detecting the clog position (sometimes more than one could be identified), the hanging arches were destroyed. To this end we employ a set of 10 electric shakers placed at the back of the pipe. From them, only the nearest to the (already identified) clog was activated for a lapse of $0.5 \mathrm{~s}$. The shaker hit several times the tube with a tuned intensity that was strong enough to break the clog. After this, the conveyor was switched on, resuming the extraction of material and the measurement loop started again. All these operations were controlled with a computer, allowing the complete automation of the experiment.

\section{Experimental results}

We start presenting the avalanche duration distribution obtained for the given set of parameters specified above: a $200 \mathrm{~cm}$ length and $2.2 \mathrm{~cm}$ inner diameter tube full of $0.6 \mathrm{~cm}$ diameter glass beads discharged at a constant velocity of $0.215 \mathrm{~cm} / \mathrm{s}$. As displayed in Fig. 2 the distribution looks like an exponential tail, in agreement with previous results obtained in silos [6-9] and for faceted particles discharged through an inclined pipe [10]. The explanation given to justify this tail in all these different works relies on the existence of a constant probability of clogging over the whole duration of the avalanche. Here, we can argue the same despite that, contrary to the silo (where clogging always happens above the outlet), in the pipe, clogging can appear at different positions.

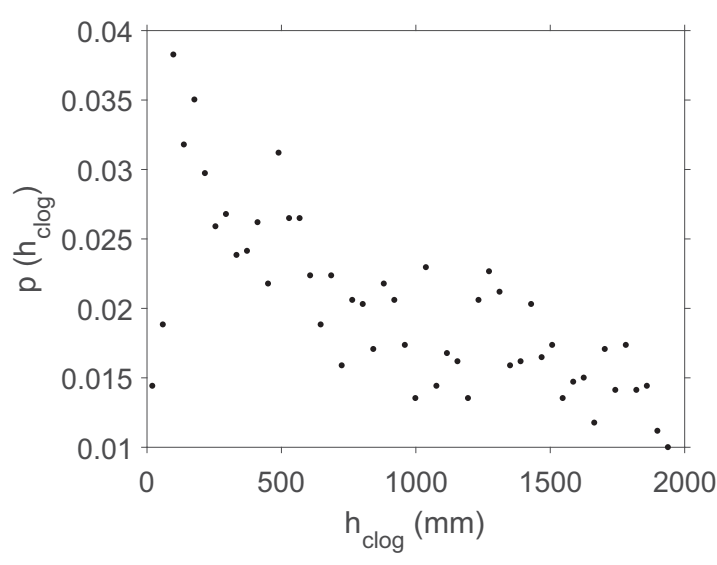

Figure 3. Probability of clogging at different positions within the pipe, measured as the height $h$ from the bottom. The probability has been obtained in 50 bins of $4 \mathrm{~cm}$ each.

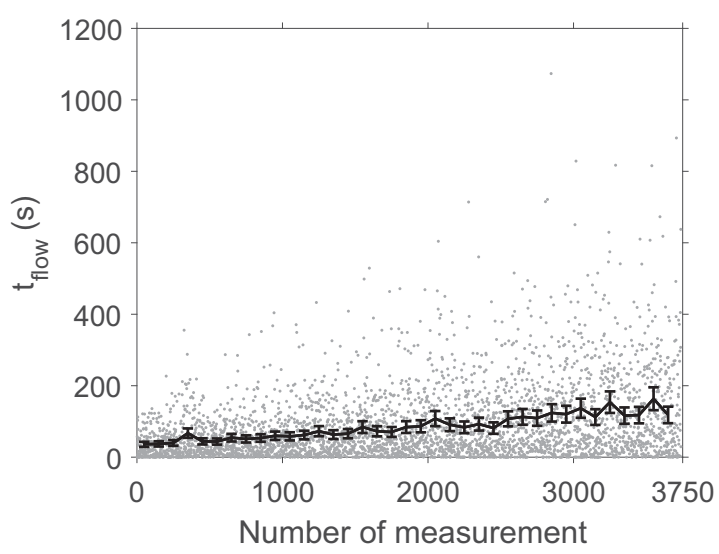

Figure 4. $t_{\text {flow }}$ versus number of measurement. Gray dots represent the experimental results of $t_{\text {flow }}$. The black line links the averages of 100 consecutive measurements of $t_{\text {flow }}$. The error bars are the $95 \%$ confidence intervals of these averages assuming an exponential distribution.

In the next stage we analyze, precisely, where the hanging clogs appear. In Fig. 3 we show the probability of finding a clog at different heights of the pipe, $h$, measured from the bottom. Clearly, if we exclude the lower $4 \mathrm{~cm}$ we can state that clogging is more frequent near the bottom and reduces monotonously with height. The decrease looks nonlinear but more statistics than the 3738 clogs obtained are needed to venture any deeper conclusion.

Up to now, we have shown that in a very long pipe full of grains, clogs are possible even when the granular material is formed by spheres. This was not trivial as in previous experimental works, faceted anisotropic particles were used, and those are known to clog more as they align each other and with the walls [12-14]. On the contrary, hanging clogs of spherical particles are necessarily stabilized by frictional forces: at some position of the arch, a bead should be hanging below the equator of their neighbours (or below the equator of a neighbour and the wall). 


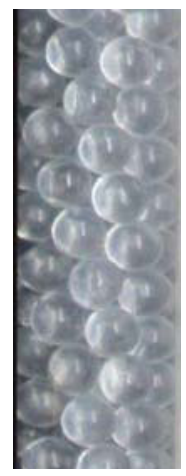

Figure 5. Helical arrangement of glass beads formed spontaneously inside the pipe.

These kind of arches have been also found in static silos and have been demonstrated to be very weak, in the sense that they are easily destabilized [15]. We believe that this arch weakness and strong dependence on frictional forces is also behind an undesirable effect that we have found in our experiments: the avalanche duration is not constant over time (at least under these experimental conditions). This is readily appreciated in Fig. 4 where we plot each avalanche duration in chronological order. The black line represents the averages for 100 consecutive avalanche durations. At this moment, we do not fully understand this effect but we believe that it could be related with the fact that, over time, the glass beads or the methacrylate tube wear out. In such case, a little amount of dust could lubricate the material and delay the formation of clogs.

\section{Conclusions}

In this work we have shown that spherical beads flowing within a vertical narrow tube are able to display clogging events provided that the tube diameter is small enough in comparison with the particles size. As previously reported, we observe that the duration of clogs displays an exponential distribution. Moreover, the experimental improvements performed to observe the position of the hanging arches allows to find a net spatial dependence of them: clogging is more frequent in the lower parts of the tube. The origin of this is not clear but it could be speculated that clogging could increase with pressure and, in such narrow tube, the pressure does not really saturates as predicted by Janssen [16]. An alternative explanation that might also affect the way in which pressure propagates within the pipe could be based in the spontaneous formation of helical patterns observed inside the tube (Fig. 5). Indeed, recent findings have shown that these helical patterns lead to clogging reduction [11]. Undoubtedly, a careful investigation of such structures and the implications that they have for pressure transmission is necessary. Interestingly, these helices seem to be generic, being the focus of interest in disparate scenarios such as in granular media aiming for packing optimization [17]; in botany concerning the ordering of the leaves of a plant (Phyllotaxis) [18]; or in the self assembly of nanospheres inside carbon nanotubes [19].

\section{Acknowledgments}

Diego López thanks Asociación de Amigos de la Universidad de Navarra for a scholarship. This work was funded by Ministerio de Economía y Competitividad (Spanish Government) through Project No. FIS2014-57325.

\section{References}

[1] W. A. Hustrulid and W. A. Hustrulid and R. C. Bullock, Underground Mining Methods: Engineering Fundamentals and International Case Studies (Society for Mining and exploration Inc., 2001) 627-634.

[2] V. Aytaman, Canadian Mining Journal 81, 77-81 (1960).

[3] J. Hadjigeorgiou, J. F. Lessard and F. MercierLangevin, Afr. Inst. Min. Metall 105, 809-816 (2005).

[4] J. Hadjigeorgiou and J. F. Lessard, Rock Mechanics and Mining Science 44, 820-834 (2007).

[5] K. To et al., Phys. Rev. Lett. 86, 71 (2001).

[6] I. Zuriguel, L. A. Pugnaloni, A. Garcimartín and D. Maza, Phys. Rev. E 68, 030301 (R) (2003).

[7] I. Zuriguel, A. Garcimartín, D. Maza, L. A. Pugnaloni and J. M. Pastor, Phys. Rev. E 71, 051303 (2005).

[8] K. To, Phys. Rev. E 71, 060301 (R) (2005).

[9] C. C. Thomas and D. J. Durian, Phys. Rev. Lett. 114, 178001 (2015).

[10] A. Janda, I. Zuriguel, A. Garcimartín and D. Maza, Granular Matter 17, 545 (2015).

[11] F. Verbücheln, E. Parteli and T. Pöschel, Soft Matter 11, 4295-4305 (2015).

[12] T. Kanzaki, M. Acevedo, I. Zuriguel, I. Pagonabarraga, D. Maza and R.C. Hidalgo. The European Physical Journal E 34, 133 (2011).

[13] J. Tang and R. P. Behringer. Europhysics Letters 114, 34002 (2016).

[14] A. Ashour, S. Wegner, T. Trittel, T. Börzsönyi, and R. Stannarius, Soft Matter 13, $402-414$ (2017).

[15] C. Lozano, G. Lumay, I. Zuriguel, R. C. Hidalgo and A. Garcimartín, Phys. Rev. Lett. 109, 068001 (2012).

[16] H. A. Janssen Versuche über Getreidedruck in Silozellen. Zeitschr. d. Vereines deutscher Ingenieure 39, (1895) 1045.

[17] A. Mughal, H. K. Chan, D. Weaire and S. Hutzler. Phys. Rev. E 85, 051305 (2012).

[18] R. Erickson, Science 181, 705-716 (1973).

[19] A Khlobystov, D. Britz, A. Ardavan and A. Briggs, Phys. Rev. Lett. 92, 245507 (2004). 